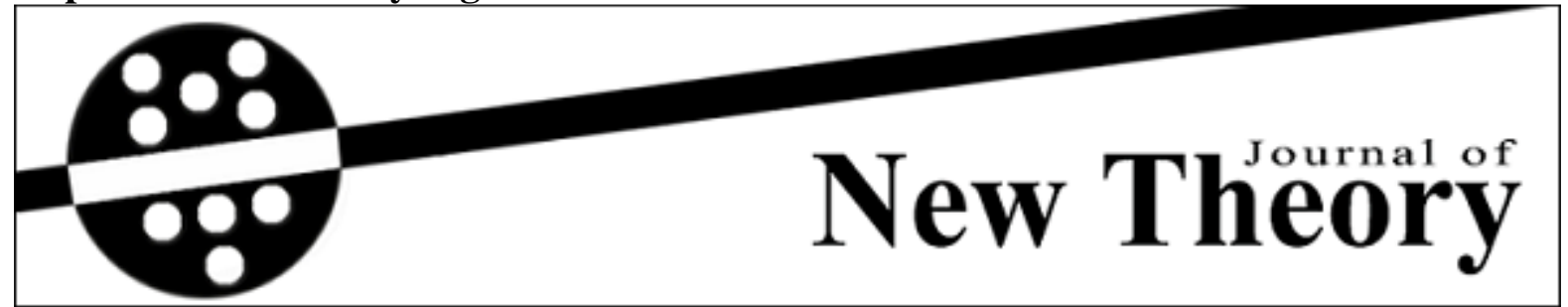

Received: 15.09 .2017

Published: 09.10.2017

Year: 2017, Number: 16, Pages: 49-51

Original Article

\title{
THE JACOBIAN CONJECTURE IS TRUE
}

Kerimbayev Rashid Konyrbayevich <ker_im@mail.ru>

Kazakh National University After al-Farabi, Mathematics Department, 050040, Almaty, Kazakhstan

\begin{abstract}
We are talking about famous the Jacobian conjecture. Let $f$ and $g$ be polynomials dependent from two variables over the field $\mathrm{K}$ zero characteristics, $f(x, y), g(x, y) \in K[x, y]$.

Keywords - Jecobian Conjecture, Polynomial Maps.
\end{abstract}

\section{Introduction}

The Jacobian conjecture consists in next: If Jacobian $J(f, g)(x, y)$ of polynomials $f, g$ are invertible in the ring $K[x, y]$, then polynomials of $f, g$ gives $K$-automorphism of the ring $K[x, y]$.

The Jacobian conjecture solved for a particular case. They are presented in the book [1]. Although, the problem does not fully solved. Below we will describe the solution of the problem.

\section{Formal Inverse Mapping}

On the line with polynomials $K[x, y]$, also important to consider and ring of formal power series $K[[x, y]]$, where $K$ - field. Here several pitfalls. Composition of polynomials are always defined, but composition of series does not. Composition of formal power series are defined in case, when power series without free terms. In other side, formal power series, different from zero with free term, always invertible in the ring $K[[x, y]]$. So, in the book [1] has proven next theorem. 
Formal inverse function theorem. Let $f(x, y), g(x, y) \in K[[x, y]]$ be formal power series with next properties:

$$
f(0,0)=0, g(0,0)=0 \text { and } J(f, g)(0,0) \in K^{*} .
$$

Then exists formal power series $u(x, y), v(x, y) \in K[[x, y]]$ such as

$$
u(0,0)=0, v(0,0)=0 \quad \text { and } u(f, g)=x, v(f, g)=y .
$$

Moreover, such formal power series unique and satisfies condition $f(u, v)=x, g(u, v)=y$. As result of the theorem immediately we can get next lemma.

Lemma. If $f(x, y), g(x, y) \in K[[x, y]]$ polynomials with properties

$$
f(0,0)=0, g(0,0)=0 \text { and } J(f, g)(x, y) \in K^{*}
$$

Then algebraic variate of polynomials $f$ and $g$ consists from one zero point. Exactly,

$$
V(f, g)=\left\{(x, y) \in K^{2} \mid f(x, y)=0, g(x, y)=0\right\}=\{(0,0)\}
$$

Proof. Indeed, by the theorem of formal inverse function, exist series

$$
u(x, y), v(x, y) \in K[[x, y]]
$$

such as

$u(0,0)=0=v(0,0)$ and $x=u(f(x, y), g(x, y)), \quad y=v(f(x, y), g(x, y))$.

Then, if

$$
f(a, b)=0=g(a, b),
$$

then,

$$
\begin{aligned}
& a=u(f(a, b), g(a, b))=u(0,0)=0, \\
& b=v(f(a, b), g(a, b))=v(0,0)=0,
\end{aligned}
$$

that is $-V(f, g)=\{(0,0)\}$.

After all, next theorem will be proven easily.

The injective function theorem. Let $f(x, y), g(x, y) \in K[x, y]$ polynomials with properties $J(f, g)(x, y) \in K^{*}$. Then polynomials mapping

$$
\phi: K^{2} \rightarrow K^{2}, \quad \phi(x, y)=(f(x, y), g(x, y))
$$

is injective. 
Proof. Let $\phi(a, b)=\phi(c, d)$. Consider next polynomials

$$
F(x, y)=f(x+a, y+b)-f(a, b), \quad G(x, y)=g(x+a, y+b)-g(a, b)
$$

Then

$$
F(0,0)=0=G(0,0) \text { and } J(F, G)(x, y) \in K^{*}
$$

By the lemma $V(F, G)=\{(0,0)\}$. We have $F(c-a, d-b)=0, G(c-a, d-b)=0$. It means

$$
(c-a, d-b) \in V(F, G)=\{(0,0)\} .
$$

It means $c=a, d=b . \phi$ injective.

\section{References}

[1] van den Essen A. Polynomial automorphisms and the Jacobian Conjecture. Birkhauser: Progress in Mathematics, 2000. 